\title{
Analysis and interpretation of quadratic models of receptive fields
}

\author{
Pietro Berkes $^{1} \&$ Laurenz Wiskott ${ }^{2}$ \\ ${ }^{1}$ Gatsby Computational Neuroscience Unit, Alexandra House, 17 Queen Square, London WC1N 3AR, UK. ${ }^{2}$ Institute for Theoretical Biology, Humboldt-Universität zu \\ Berlin, Invalidenstraße 43, D-10115 Berlin, Germany. Correspondence should be addressed to P.B. (berkes@gatsby.ucl.ac.uk).
}

Published online 8 March 2007; doi:10.1038/nprot.2007.27

\begin{abstract}
In this protocol, we present a procedure to analyze and visualize models of neuronal input-output functions that have a quadratic, a linear and a constant term, to determine their overall behavior. The suggested interpretations are close to those given by physiological studies of neurons, making the proposed methods particularly suitable for the analysis of receptive fields resulting from physiological measurements or model simulations.
\end{abstract}

\section{INTRODUCTION}

Research in neuroscience has seen a recent trend toward the extension of receptive field (RF) estimation techniques and theoretical principles from linear to non-linear models. This development has led to the need for new tools to interpret and visualize non-linear functions. This protocol presents a number of methods that were developed, in the context of a computational model of the visual cortex ${ }^{1,2}$, to analyze quadratic forms as neuronal RFs.

Quadratic forms are used in experimental studies as quadratic approximations to the input-output function of neurons and can be derived from neural data as Volterra/Wiener approximations up to the second order ${ }^{3-13}$. In addition, several theoretical studies have defined quadratic models of neuronal RFs either explicitly $2,14,15$ or implicitly as neural networks ${ }^{16-18}$. This choice is justified by the fact that quadratic forms constitute a computationally rich function space that contains interesting elements (e.g., the standard energy model of complex cells ${ }^{19}$ ) while still having a reasonably small number of parameters. This protocol does not present a new technique to estimate neuronal RFs, but rather a procedure to analyze the estimation performed by studies such as those cited above. To illustrate the proposed methods, we make use of the results of the theoretical model presented in refs. 1 and 2 (see ANTICIPATED RESULTS).

We write quadratic forms, here also referred to as 'units', in vector notation as

$$
g(\mathbf{x})=\underbrace{\frac{1}{2} \mathbf{x}^{\mathrm{T}} \mathbf{H} \mathbf{x}}_{\text {quadratic term }}+\underbrace{\mathbf{f}^{\mathrm{T}} \mathbf{x}}_{\text {linear term }}+c,
$$

where $\mathbf{x}$ is an $N$-dimensional input vector, $\mathbf{H}$ a symmetric $N \times N$ matrix, $\mathbf{f}$ an $N$-dimensional vector and $c$ a constant. Such a quadratic form is called 'inhomogeneous' because it contains a linear and a constant term. We will also consider the simpler homogeneous case with $\mathbf{f}=\mathbf{0}$. In the model system presented in this paper, the input $\mathbf{x}$ is, for example, an image patch reshaped as a vector.

After some introductory remarks in Step 1, the second step in the protocol provides a generic visualization of a quadratic form in terms of its eigenvector decomposition. This step is interesting as a reference because it has often been used in previous studies in combination with a statistical analysis of the significance of the eigenvalues (see Step 7). However, the outcome is, in general, difficult to interpret, and it ignores the contribution of the linear term.

In Step 3, we compute the 'optimal excitatory' and 'optimal inhibitory stimulus' of a unit. This is equivalent to the physiological characterization of a neuron in terms of the stimulus to which it responds the most or by which it is most inhibited, respectively. The optimal excitatory stimulus can be subsequently used to compute the preferred stimulus parameters. For instance, in experiments concerning the visual system, one could compute the position and size of the RF and its preferred orientation and frequency. This information could then be used in successive experiments with sine gratings.

In a linear model, the optimal excitatory stimulus would give a complete description of the RF. As a quadratic form is non-linear, the optimal stimuli give only partial information about the response properties. Additional information can be obtained by computing the invariances at the optimal stimulus, i.e., the transformations of the stimuli to which the function is most invariant (Step 4). Invariances are a common concept in physiology: neurons are thought to respond strongly to a given stimulus but to be insensitive to changes in some of its properties. For example, complex cells in the primary $\mathrm{V} 1$ are commonly described as being optimally responsive to a sine grating of a particular frequency and orientation while being insensitive to its phase. The following three steps (Steps 5-7) are used to visualize the invariances and to determine which of them are statistically significant.

The detailed mathematical derivation of the equations used in this protocol can be found in ref. 20, and the mathematical terms used in the procedure are defined in Table 1. This article focuses on the practical use of the algorithms and on their implementation.

\section{MATERIALS}

A Matlab implementation of the algorithms described in this protocol (Boxes 1 and 2) is available at http://www.gatsby.ucl.ac.uk/ berkes/ software/qforms-tk/. The algorithms are also part of the MDP
Python library (Berkes, P. and Zito, T., Modular Toolkit for Data Processing (version 2.0), http://mdp-toolkit.sourceforge.net, 2006). 
TABLE 1 | Definition of mathematical terms.

\begin{tabular}{ll}
\hline$N$ & Dimensionality of the input space \\
$g$ & The inhomogeneous quadratic form \\
$\mathbf{H}, \mathbf{h}_{i}$ & $N \times N$ matrix of the quadratic term of $g$ and the $i$-th row of $\mathbf{H}$. $\mathbf{H}$ is assumed to be symmetric \\
$\mathbf{V}_{i}, \mu_{i}$ & The $i$-th eigenvector and eigenvalue of $\mathbf{H}$, sorted by decreasing eigenvalues (i.e., $\left.\mu_{1} \geq \mu_{2} \geq \ldots \geq \mu_{N}\right)$ \\
$\mathbf{V}, \mathbf{D}$ & Matrix of the eigenvectors $\mathbf{V}=\left(\mathbf{v}_{1}, \ldots \mathbf{v}_{N}\right)$ and diagonal matrix of the eigenvalues, so that $\mathbf{V}^{\top} \mathbf{H V}=\mathbf{D}$ \\
$\mathbf{f}$ & $\quad N$-dimensional vector of the linear term of $g$ \\
$\mathbf{C}$ & Scalar value of the constant term of $g$ \\
$\mathbf{x}^{+}, \mathbf{x}^{-}$ & Optimal excitatory and inhibitory stimuli, $\left\|\mathbf{x}^{+}\right\|=\left\|\mathbf{x}^{-}\right\|=r$
\end{tabular}

\section{PROCEDURE}

1| Normalize the quadratic form. The analysis starts with a given quadratic form provided, for instance, by a model simulation or a physiological experiment. Without loss of generality, we assume that the quadratic form is normalized such that matrix $\mathbf{H}$ is symmetric, the neutral stimulus (usually an average or blank stimulus) is zero, and the response to the neutral stimulus is zero as well, so that positive responses are excitatory and negative ones are inhibitory. This can be easily achieved by shifting the coordinate systems and by making $\mathbf{H}$ symmetric. If $\mathbf{x}_{0}^{\prime}$ indicates the actual, possibly non-zero, neutral stimulus and

$$
g^{\prime}\left(\mathbf{x}^{\prime}\right)=\frac{1}{2} \mathbf{x}^{\prime \top} \mathbf{H}^{\prime} \mathbf{x}^{\prime}+\mathbf{f}^{\prime \top} \mathbf{x}^{\prime}+C^{\prime}
$$

is the original quadratic form with a possibly non-symmetric $\mathbf{H}^{\prime}$, then shifting the input coordinate system by $-\mathbf{x}_{0}^{\prime}$ and the output coordinate system by $-g^{\prime}\left(\mathbf{x}_{0}^{\prime}\right)$ yields the required normalized form

$$
\begin{aligned}
g(\mathbf{x}): & =g^{\prime}\left(\mathbf{x}+\mathbf{x}_{0}^{\prime}\right)-g^{\prime}\left(\mathbf{x}_{0}^{\prime}\right) \\
& =\frac{1}{2}\left(\mathbf{x}+\mathbf{x}_{0}^{\prime}\right)^{\top} \mathbf{H}^{\prime}\left(\mathbf{x}+\mathbf{x}_{0}^{\prime}\right)+\mathbf{f}^{\prime \top}\left(\mathbf{x}+\mathbf{x}_{0}^{\prime}\right)+c^{\prime}-g^{\prime}\left(\mathbf{x}_{0}^{\prime}\right), \\
& =\frac{1}{2} \mathbf{x}^{\top} \mathbf{H} \mathbf{x}+\mathbf{f}^{\top} \mathbf{x}
\end{aligned}
$$

with

$$
\begin{aligned}
\mathbf{H} & :=\frac{1}{2}\left(\mathbf{H}^{\prime}+\mathbf{H}^{\prime \top}\right), \\
\mathbf{f} & :=\mathbf{H} \mathbf{x}_{0}^{\prime}+\mathbf{f}^{\prime},
\end{aligned}
$$

where $\mathbf{H}$ now has the required symmetry. Given a stimulus $\mathbf{x}$ or a response $g(\mathbf{x})$ in the normalized coordinate system, the input or output in the original space is recovered simply by adding $\mathbf{x}_{0}^{\prime}$ or $g^{\prime}\left(\mathbf{x}_{0}^{\prime}\right)$, respectively. Although after normalization the constant term falls off, in the rest of the protocol we are going to provide results for general quadratic forms with $c \neq 0$, which might be useful in some particular situations.

2| Visualize the eigenvectors of $\mathbf{H}$ in descending order. This optional step is given for comparison with the conventional analysis of quadratic models of RFs, which comprises the visualization of the eigenvectors $\mathbf{v}_{1}, \ldots, \mathbf{v}_{N}$ of the quadratic term $\mathbf{H}$, sorted by decreasing eigenvalues $\mu_{1} \geq \mu_{2} \geq \cdots \geq \mu_{N}$. If the input data have a 2D spatial arrangement (e.g., in case of image patches), the eigenvectors can be visualized as a series of patches by reshaping the vectors $\mathbf{v}_{i}$ to match the structure of the input (see Fig. 1). Eigenvectors corresponding to

\section{BOX 1 | PSEUDOCODE OF THE ALGORITHM THAT COMPUTES THE OPTIMAL EXCITATORY STIMULUS OF THE INHOMOGENEOUS QUADRATIC FORM (STEP 3)}

In the following code, $\mathbf{A}$ ' means 'A transposed'. The algorithm can be used to compute the optimal inhibitory stimulus by applying it to the negative of the quadratic form.

input: $\quad H, f, c:$ quadratic form r: norm of the solution ( \pm eps) eps: tolerance of norm (x) from $r$

output: $\quad x \_m a x$ : optimal excitatory stimulus $x+$

\# compute the eigenvalues and eigenvectors of $\mathrm{H}$

1- $\quad \mathrm{mu}(1), \ldots, \mathrm{mu}(\mathrm{N}):=$ eigenvalues(H)

2- $\quad \mathrm{v}(1), \ldots, \mathrm{v}(\mathrm{N}):=$ eigenvectors $(\mathrm{H})$ \# compute the coefficients of the eigenvector \# decomposition of $f$ (eqns. 3.3, 3.4)

3- $\quad$ alpha(i) $:=v(i)^{\prime *} f$ \# compute the range of the parameter lambda

4- lambda_left $:=\max (\mathrm{mu})$

5- $\quad$ lambda_right $:=\operatorname{norm}(\mathrm{f}) / \mathrm{r}+\max (\mathrm{mu})$

\# search by bisection until norm $(x)^{\wedge} 2=r^{\wedge} 2$

\# norm_x_2 holds the value of norm $(x)^{\wedge} 2$

\# at the current lambda

6- norm_x_2:=0

7- $\quad$ while abs(sqrt(norm_x_2)-r) > eps:

\# bisect the interval

8- $\quad$ lambda $=($ lambda_right-lambda_left $) / 2+$ lambda_left \# compute the eigenvalues of $(\text { lambda* } \mathbf{I}-\mathrm{H})^{\wedge}-1$ beta(i) $:=1 /($ lambda-mu(i))

\# compute norm $(x)^{\wedge} 2$ at lambda (eqn. 3.4)

norm_ $\times 2=\operatorname{sum}\left(\operatorname{beta}(i)^{\wedge} 2\right.$ * alpha $\left.(i)^{\wedge} 2\right)$

\# update the interval limits

if norm_x_2 $>r^{\wedge} 2$ :

lambda_left $=$ lambda

else:

lambda_right $=$ lambda

\# lambda found, compute the solution (eqn. 3.3)

15- $\quad x \_m a x=\operatorname{sum}\left(\operatorname{beta}(i)^{*} v(i) * a l p h a(i)\right)$ 
PROTOCOL
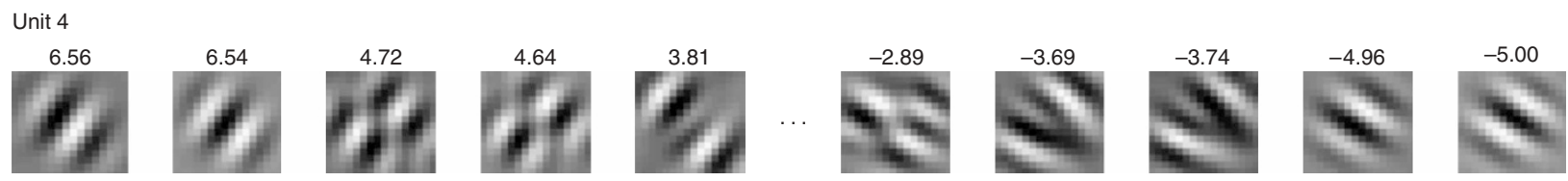

Unit 28
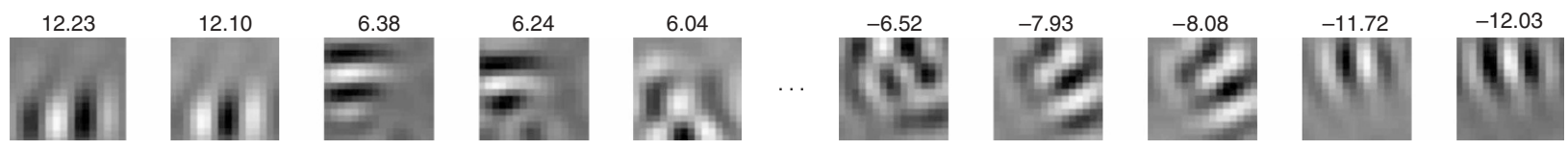

Figure 1 | Eigenvectors of the quadratic term of two units learned in the simulation. The corresponding eigenvalues are indicated above the eigenvectors.

positive or negative eigenvalues give excitatory and inhibitory contributions, respectively, as the quadratic term can be rewritten as

$$
\frac{1}{2} \mathbf{x}^{\top} \mathbf{H} \mathbf{x}=\frac{1}{2} \sum_{i=1}^{N} \mu_{i}\left(\mathbf{v}_{i}^{\top} \mathbf{x}\right)^{2} .
$$

Stimuli belonging to the space spanned by the eigenvectors with largest positive eigenvalues (i.e., all stimuli formed by a linear combination $\mathbf{x}=a_{1} \mathbf{v}_{1}+\cdots a_{K} \mathbf{v}_{K}$ of those eigenvectors) are thus going to elicit a strong response in the unit, and, conversely, points in the space spanned by those eigenvectors with largest negative eigenvalues are going to strongly inhibit the unit. This form of visualization has several problems. First, although the first and the last eigenvector typically have a very clear structure that is easy to interpret, the next ones very quickly look unstructured and do not lend themselves to an intuitive interpretation. Second, assume we are interested in the set of stimuli that yield a strong response of, say, more than $80 \%$ of the maximum response (given a certain stimulus energy). Some of the eigenvectors that would be discarded because they yield a response of less than $80 \%$ could still be necessary to generate stimuli that yield such a strong response (see Step 4 in the homogeneous case). Third, this method does not take into account the contribution of the linear term, which cannot generally be neglected ${ }^{5}$. The following steps describe an alternative method based on optimal stimuli and their invariances that does not suffer from these problems.

3| Compute the optimal stimuli $\mathbf{x}^{+}$and $\mathbf{x}^{-}$. The optimal excitatory stimulus $\mathbf{x}^{+}$(also called the 'preferred stimulus' in the literature) is defined as the input vector that maximizes the output of the model neuron given a fixed energy $\left\|\mathbf{x}^{+}\right\|=\mathbf{r}$. The fixed energy constraint is necessary to make the problem well posed. Without such a constraint, arbitrarily high outputs can be generated if only the input is large enough in amplitude and points in a direction where the quadratic term is positive. This constraint is also the reason the quadratic form should be defined such that $\mathbf{x}=0$ is the neutral stimulus relative to which stimulus energy is measured. (As an alternative one could use the constraint $\left\|\mathbf{x}-\mathbf{x}_{0}^{\prime}\right\|=r$, with $\mathbf{x}_{0}^{\prime}$ indicating the neutral stimulus, but this would clutter the analysis.) The optimal stimulus is thus defined by the following equations:

Maximize

$$
g(\mathbf{x})=\frac{1}{2} \mathbf{x}^{\top} \mathbf{H} \mathbf{x}+\mathbf{f}^{\top} \mathbf{x}+\boldsymbol{C}
$$

under the constraint

$$
\|\mathbf{x}\|=r \text {. }
$$

It can be shown ${ }^{20}$ that the solution is unique in general and has the form $\mathbf{x}=(\lambda \mathbf{I}-\mathbf{H})^{-1} \mathbf{f}$, where $\lambda$ is a scalar between $\mu_{1}$ and $\frac{\|\mathbf{f}\|}{r}+\mu_{1}$. The solution can therefore be found by searching over $\lambda$ until Constraint 3.2 is satisfied.

The vector $\mathbf{x}$ and norm $\|\mathbf{x}\|$ can be efficiently computed for each $\lambda$ using the expressions

$$
\mathbf{x}=\sum_{i} \frac{1}{\lambda-\mu_{i}} \mathbf{v}_{i}\left(\mathbf{v}_{i}^{\top} \mathbf{f}\right)
$$

and

$$
\|\mathbf{x}\|^{2}=\sum_{i}\left(\frac{1}{\lambda-\mu_{i}}\right)^{2}\left(\mathbf{v}_{i}^{\top} \mathbf{f}\right)^{2}
$$

The terms $\mathbf{v}_{\mathbf{i}}\left(\mathbf{v}_{i}^{\top} \mathbf{f}\right)$ and $\left(\mathbf{v}_{i}^{\top} \mathbf{f}\right)^{2}$ are constant for a given quadratic form and can be computed in advance. As the norm of $\mathbf{x}$ is monotonically decreasing in the considered interval (Equation 3.4), the search can be performed efficiently using a simple bisection method. The pseudocode yielding the desired result is shown in Box 1. In the same way, it is possible to compute the optimal 
inhibitory stimulus $\mathbf{x}^{-}$by minimizing Equation (3.1), which is equivalent to maximizing $-g(\mathbf{x}) \cdot \mathbf{x}^{-}$is the stimulus that most effectively inhibits the response of the unit.

Figure 2 shows optimal stimuli for units from the model simulation. If the quadratic form is homogeneous, the optimal excitatory stimulus points in the direction of the first eigenvector and is given by

$$
\mathbf{x}^{+}= \pm r \mathbf{v}_{1} \text {. }
$$

In the same way, the optimal inhibitory stimulus is given by $\mathbf{x}^{-}= \pm r \mathbf{v}_{N}$. This is one of the cases where the optimal stimuli are not unique but determined only up to the sign. If in addition the first eigenvalues were equal, any normalized linear combination of the corresponding eigenvectors would be an optimal stimulus.

4| Compute the invariances at $\mathbf{x}^{+}$and $\mathbf{x}^{-}$. The optimal excitatory and inhibitory stimuli give us a first impression of the response properties of a unit and two anchor points at which we can refine the analysis. In this step we compute the invariances at the optimal stimuli, i.e., the transformations of $\mathbf{x}^{+}$ and $\mathbf{x}^{-}$to which the unit is most invariant. As in Step 3, we consider inputs of constant energy $r$, because otherwise a change in the response as we vary the direction of $\mathbf{x}$ around $\mathbf{x}^{+}$ could always be compensated by a change of length (i.e., energy) of $\mathbf{x}$. Notice that around $\mathbf{x}^{+}$the response can only drop and around $\mathbf{x}^{-}$it can only rise, because the optimal stimuli are extremal points. Mathematically we have to derive the quadratic form of $g(\mathbf{x})$ constrained to the tangent space of the constant-energy sphere at $\mathbf{x}^{+}$or $\mathbf{x}^{-}$and find the directions of smallest second derivative. In the following, we give the procedure for $\mathbf{x}^{+} ; \mathbf{x}^{-}$can be treated analogously:

(i) Apply the Gram-Schmidt orthogonalization algorithm to the vectors $\mathbf{x}^{+}, \mathbf{e}_{2}, \mathbf{e}_{3}, \ldots, \mathbf{e}_{\mathrm{N}}$, where $\mathbf{e}_{j}$ is an element of the canonical basis; i.e., the $i$-th element of $\mathbf{e}_{j}$ is one and the rest is zero (a simple implementation of this algorithm is included in the Matlab library, see MATERIALS). As a result, we obtain a basis $\mathbf{b}_{1}, \ldots, \mathbf{b}_{N}$, where $\mathbf{b}_{1}=\frac{1}{r} \mathbf{x}^{+}$and $\mathbf{b}_{2}, \ldots, \mathbf{b}_{N}$ are vectors orthogonal to it, which therefore form a basis of the space tangential to the sphere of radius $r$ in $\mathbf{x}^{+}$. By restricting our computations to that space we locally enforce the constant energy constraint $\|\mathbf{x}\|=\mathrm{r}$. Define $\mathbf{B}:=\left(\mathbf{b}_{2}, \ldots, \mathbf{b}_{N}\right)^{\top}$.

(ii) Compute the directions of the invariances, which are given by the eigenvectors $\tilde{\mathbf{v}}_{i}$ of $\tilde{\mathbf{H}}:=\mathbf{B}^{\top} \mathbf{H B}$, ordered by decreasing eigenvalues $v_{1} \geq \cdots \geq v_{N-1}$. Project the eigenvectors back to the input space with

$$
\mathbf{w}_{i}=\mathbf{B} \tilde{\mathbf{v}}_{i} \text {. }
$$

The second derivative in the direction of $\mathbf{w}_{i}$ corresponds to the rate of change in the output caused by an infinitesimal movement from $\mathbf{x}^{+}$in the $\mathbf{w}_{i}$ direction. A small second derivative thus corresponds to a strong invariance. It is given by

$$
v_{i}-\frac{1}{r^{2}}\left(\mathbf{x}^{+\top} \mathbf{H} \mathbf{x}^{+}+\mathbf{f}^{\top} \mathbf{x}^{+}\right),
$$

BOX 2 | PSEUDOCODE OF THE ALGORITHM THAT COMPUTES AND VISUALIZES THE INVARIANCES OF A QUADRATIC FORM AT $\mathrm{X}^{+}$(STEPS 4 AND 5)

\author{
In the following code, $\mathbf{A}^{\prime}$ means ' $\mathbf{A}$ transposed'. \\ Input: $\quad H, f, c$ : quadratic form \\ x_max: optimal excitatory stimulus $\mathrm{x}^{+}$ \\ dalpha: precision (angular step in degrees on the \\ sphere for each frame of the animation)
}

Output: $w(1), \ldots, w(N-1)$ : directions of the invariances, sorted by increasing magnitude of the second derivative

$\mathrm{nu}(1), \ldots, \mathrm{nu}(\mathrm{N}-1)$ : value of the second derivative in the directions $w(1), \ldots, w(N-1)$

\section{\# determine the radius of the sphere}

1- $\quad r:=$ norm(x_max)

\# find a basis for the tangential plane of the sphere $\#$ in $x+; e(1), \ldots, e(N)$ is the canonical basis \# for $\mathbf{R}^{\wedge} \mathrm{N}$

$b(1), \ldots, b(N):=x \_m a x, e(2), \ldots, e(N)$

$b(1), \ldots, b(N):=$ orthogonalize $(b(1), \ldots, b(N))$

\# after orthogonalization, $b(1)=x \_\max / r$

\# restrict the matrix $\mathrm{H}$ to the tangential plane

$B:=$ matrix with $b(2), \ldots, b(N)$ as columns

$\mathrm{Ht}:=\mathrm{B}^{\prime *} \mathrm{H}^{*} \mathrm{~B}$

\# compute the eigenvalues (in decreasing order)

$\#$ and the eigenvectors

6- $\quad \mathrm{nu}(1), \ldots, \mathrm{nu}(\mathrm{N}):=$ eigenvalues $(\mathrm{Ht})$

7- $\quad \mathrm{w}(1), \ldots, \mathrm{w}(\mathrm{N}):=$ eigenvectors $(\mathrm{Ht})$

\# compute the second derivative in the direction of \# the eigenvectors (eqn. 4.2)

8- $\quad$ nu(i) $:=n u(i)-1 / r^{\wedge} 2 *\left(x \_m a x^{\prime *} H^{*} x \_m a x+f^{\prime *} x \_m a x\right)$ \# project the eigenvectors back to $R^{\wedge} N$ (eqn. 4.1)

9- $\quad w(i):=B^{*} w(i)$

\# compute the value of the quadratic form at the maximum

10- out0 $:=0.5^{*} x \_m a x^{\prime *} H^{*} x \_m a x+f^{\prime *} x \_m a x+c$ \# minimal threshold value ( 80 percent of the maximum)

11- minout $:=0.8^{*}$ out 0

\# visualize the invariances (eqn. 5.1)

12- for i from 1 to $\mathrm{N}-1$ :

13- out $:=$ out0

14- alpha $:=0$

15- $\quad x:=x \_m a x$

16- $\quad$ while out $>$ minout:

17- visualize $x$

18- alpha $:=$ alpha + dalpha

$19-$

20-

21-

which is non-positive, since $\mathbf{x}^{+}$is a local maximum. The first

term corresponds to the second derivative within the tangent space; the second term is a correction term, equal for all invariances, resulting from the fact that the movement is constrained to the surface of the sphere of constant energy (see ref. 20). 
PROTOCOL

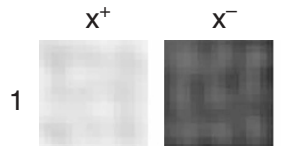

6

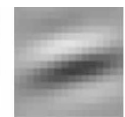

11
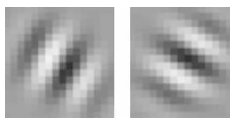

26

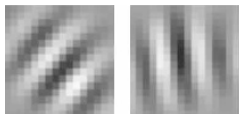

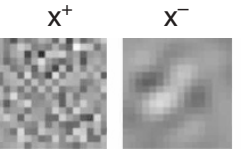
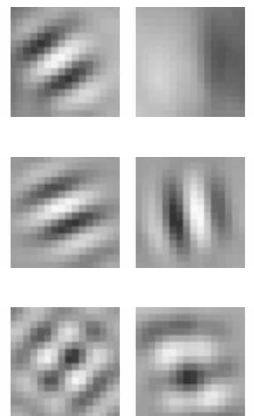
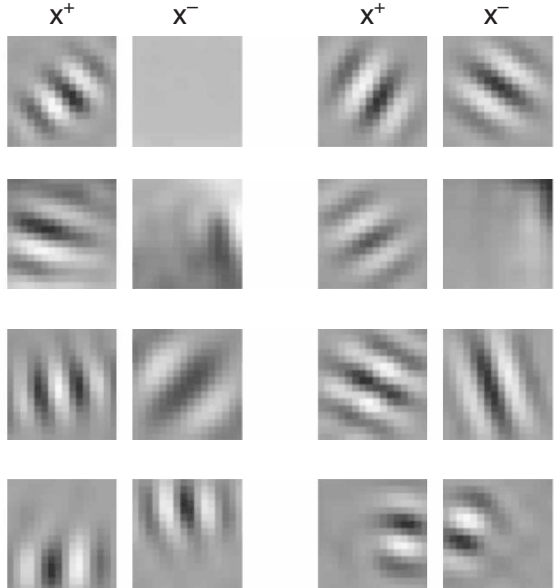

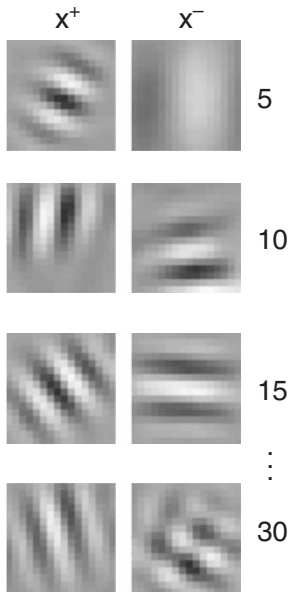

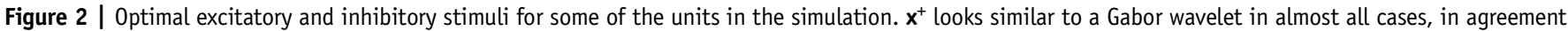
with physiological data. $\mathbf{x}^{-}$is usually structured and similar to a Gabor wavelet as well, which suggests that inhibition plays an important role. Units are numbered consecutively from 1 to 15 in the top four rows and from 26 to 30 in the last row.

In the next step, we describe how to visualize the invariances, and in Steps 6 and 7 we discuss how to check which invariances are significant.

If the quadratic form is homogeneous, the invariances at $\mathbf{x}^{+}$are given by the eigenvectors $\mathbf{v}_{2}, \ldots, \mathbf{v}_{N}$ in this order, and the second derivative on the sphere in the direction of $\mathbf{v}_{i}$ is $\mu_{i}-\mu_{1}$. The invariances at $\mathbf{x}^{-}$are given by $\mathbf{v}_{N-1}, \ldots, \mathbf{v}_{2}$, with second derivatives $\mu_{i}-\mu_{N}$.

5| Visualize the significant invariances. To visualize invariance $i$ in an animation, move the stimulus $\mathbf{x}$ around the optimal stimulus $\mathbf{x}^{+}$(or $\mathbf{x}^{-}$) on the sphere of constant energy in the direction of $\mathbf{w}_{i}$ along the path defined as

$$
\mathbf{x}(\alpha)=\cos (\alpha) \cdot \mathbf{x}^{+}+\sin (\alpha) \cdot r \mathbf{w}_{i} \quad \alpha \in[-90,90] .
$$

We recommend animating $\mathbf{x}(\alpha)$ only within the range of $\alpha$ where the response maintains at least $80 \%$ of the maximal response (Fig. 3). Figure 4 shows three frames of such animations for six different invariances. The value of $\alpha$ at the extrema provides an indication of how robust the unit is to changes in that direction.

6| Collect random quadratic forms. To assess which of the invariances computed in the preceding step are statistically significant, we compare them to those of random quadratic forms with similar output statistics, which are generated in this step. Perform Option (A) for theoretical simulations and Option (B) for physiological experiments.

\section{(A) In theoretical simulations}

In many theoretical studies, one enforces a fixed mean and variance on the output of the units. Without loss of generality, we assume here zero mean and unit variance, which can be achieved as follows:

(i) Expand the input data in the space of polynomials of degree two using the mapping

$$
\Phi(\mathbf{x})=\left(x_{1} x_{1}, x_{1} x_{2}, x_{1} x_{3}, \ldots, x_{N} x_{N}, x_{1}, \ldots, x_{N}\right)^{T} .
$$

(ii) Compute the mean of the expanded data

$$
\bar{\Phi}:=\sum_{t} \Phi\left(\mathbf{x}_{t}\right),
$$

and the eigenvalue diagonal matrix $\Lambda$ and eigenvector matrix $\mathbf{E}$ of the covariance matrix

$$
\sum_{t}\left(\Phi\left(\mathbf{x}_{t}\right)-\bar{\Phi}\right)\left(\Phi\left(\mathbf{x}_{t}\right)-\bar{\Phi}\right)^{\top}
$$

Figure 3 | Illustration of the method used to visualize the invariances. Starting from the optimal stimulus (top) we move on the sphere in the direction of an invariance until the response of the unit drops below $80 \%$ of the maximal output or reaches $90^{\circ}$. In the figure two invariances of Unit 4 are visualized. The one on the left represents a phase-shift invariance and preserves more than $80 \%$ of the maximal output until $90^{\circ}$ (the output at $90^{\circ}$ is $99.6 \%$ of the maximum). The one on the right represents an invariance to orientation change. The output drops below $80 \%$ at $55^{\circ}$.

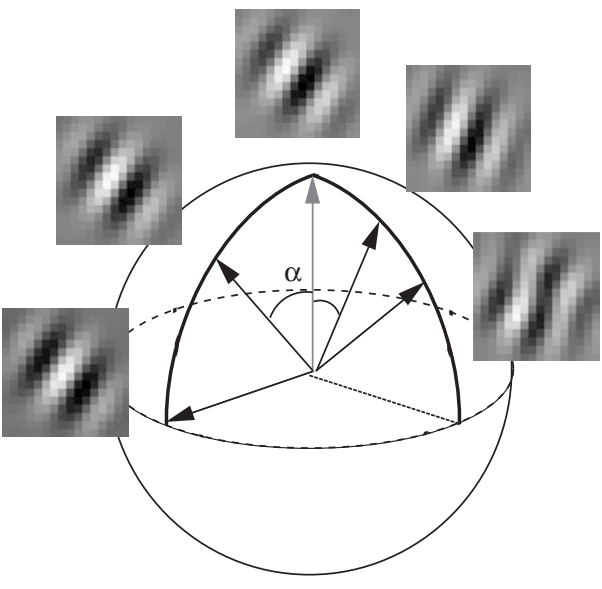


Figure 4 | Example invariances at the optimal stimuli for some of the units. The central patch of each plot represents the optimal stimulus for a unit, and the ones on the sides are produced by moving it in one (left path) or the other (right patch) direction of the eigenvector corresponding to the invariance. To produce this plot, we stopped before the output dropped below $80 \%$ of the maximum to make the interpretation of the invariances easier. The relative output of the function as a percentage and the angle of displacement $\alpha$ (eqn. 5.1) are given above the patches. The animations corresponding to these invariances are available at the authors' homepages.

(iii) Compute the whitening matrix $\mathbf{S}=\Lambda^{-1 / 2} \mathbf{E}^{\top}$.

(iv) Generate random vectors $\mathbf{q}_{i}^{\prime}$ of length 1 in the whitened, expanded space and derive the corresponding quadratic forms in the original input space using the relation

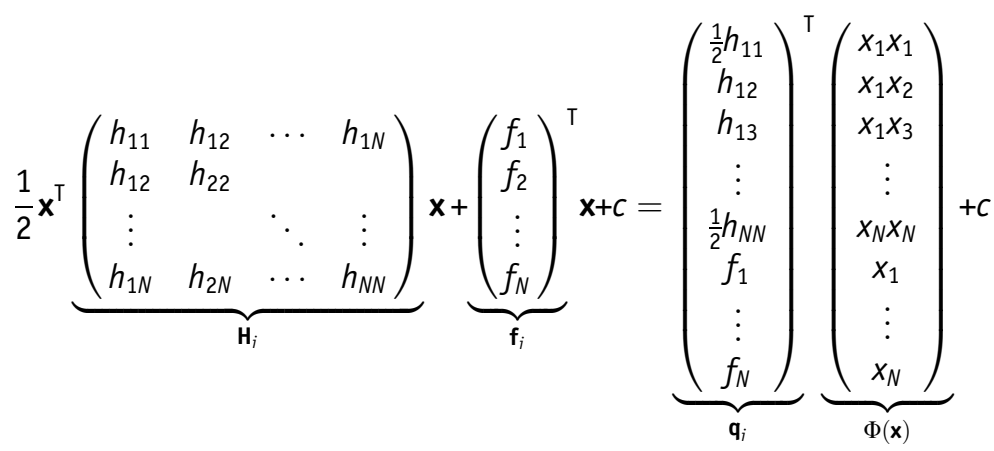

with $\mathbf{q}_{i}:=\mathbf{S}^{\top} \mathbf{q}_{i}^{\prime}$ and $c:=\mathbf{q}_{i}^{\top} \bar{\Phi}$. The output of the resulting random quadratic forms has zero mean and unit variance by construction.

$\triangle$ CRITICAL STEP The random vectors of length 1 must be uniformly distributed on the unit sphere. This can be easily achieved by repeatedly drawing random numbers from a zero-mean Gaussian distribution, arranging them in a vector and then normalizing the vector to norm 1 . It is important not to use random numbers from a uniform distribution, because that would introduce a strong bias toward the corners of the unit cube.

\section{(B) In physiological experiments}

(i) In physiological experiments, random quadratic forms can be generated by bootstrapping. The spikes can be shuffled ${ }^{6,10}$ or the entire spike train can be shifted relative to the stimulus sequence ${ }^{9,12}$ (this second possibility is to be preferred if the input stimuli have a temporal structure, i.e., a non-zero autocorrelation) and the same RF estimation procedure used to generate the units under consideration can be applied to the resulting data. The new randomly generated quadratic forms are compatible with the distribution of the input data and with the total number of spikes elicited in the neuron under consideration.

7| Compute confidence interval. Estimating a confidence interval for the invariances is difficult because of their interdependencies (we have an ordered set of invariances and not independent invariances) and because it is not clear what a good model of the background distribution is against which significance can be judged. We have, therefore, adopted a rather heuristic approach, as follows ${ }^{20}$. Compute the optimal stimuli and second derivatives of the invariances of the random quadratic forms as in Steps 3 and 4. Keep one randomly chosen second derivative per quadratic form to ensure the measurements are independent. This allows us to determine a distribution over the random second derivatives and a corresponding $95 \%$ confidence interval, which yields a significance threshold for the second derivatives of the learned units. Figure $\mathbf{5}$ shows that, in the model simulation, this threshold coincides with the value at which the distribution of the learned second derivatives changes from a rather Gaussian distribution on the left to a more structured distribution on the right, which we take as an additional hint that the threshold is reasonable. 
a

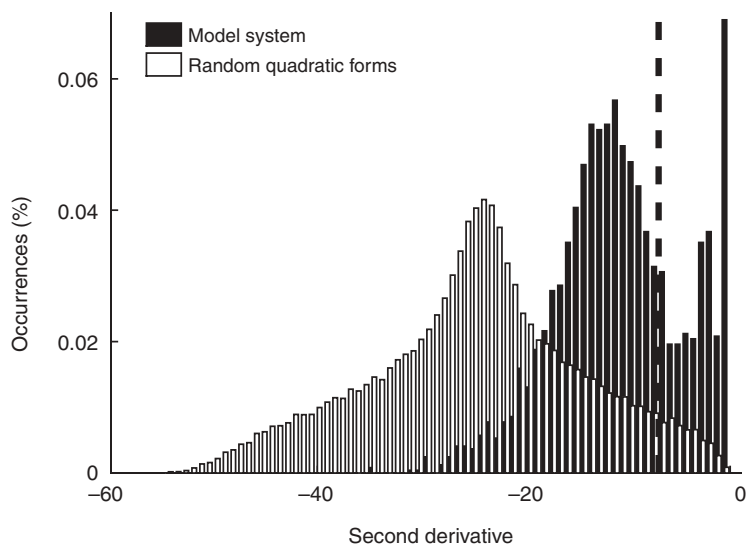

b

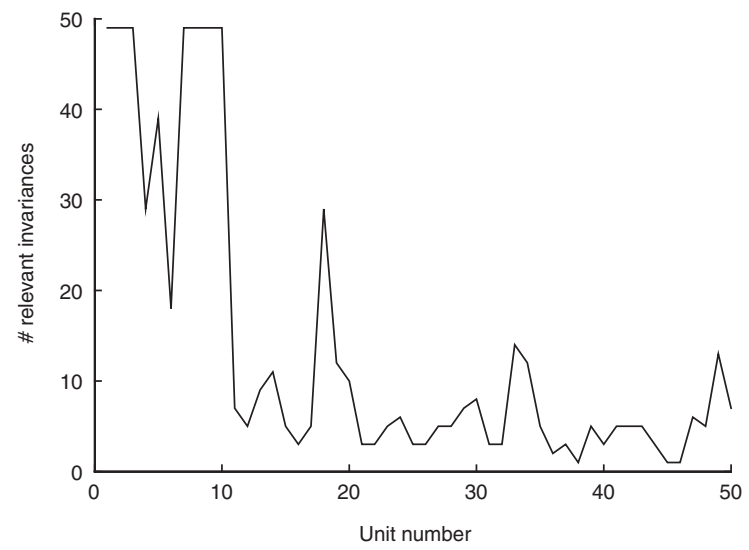

Figure 5 | Significant invariances. (a) Distribution of 50,000 independently drawn second derivatives of the invariances of random quadratic forms and distribution of the second derivatives of all invariances of the first 50 units in the simulation. The dashed line indicates the $95 \%$ confidence limit. 0 all invariances, $28 \%$ were classified as significant. (b) Number of significant invariances for each of the first 50 units learned in the simulation (the units are sorted by decreasing slowness).

\section{ANTICIPATED RESULTS}

In the following, we present the outcome of the analysis procedure described above when applied to the results of the model simulation reported in ref. 1. In that study, we applied a computational model of V1 based on the temporal slowness principle ${ }^{21}$ to natural-image-patch sequences, which yielded a set of quadratic forms representing non-linear RFs of visual neurons. The resulting units had the primary characteristics of complex cells in V1, namely, Gabor-like optimal stimuli and phase-shift invariance, as well as a number of secondary behaviors, such as end and side inhibition, direction selectivity and nonorthogonal inhibition. The results of another, more detailed simulation have been reported elsewhere? ${ }^{2}$.

\section{Step 2}

The eigenvectors of Units 4 and 28 are shown in Figure 1, ordered by decreasing eigenvalues. In Unit 4, the first two eigenvectors look similar to Gabor wavelets with a $90^{\circ}$ phase difference. As the two eigenvalues have almost the same magnitude, the response of the quadratic term is similar for the two eigenvectors and also for linear combinations thereof with constant norm 1 . For this reason, this unit responds strongly to edges or gratings but is invariant to changes in the phase or exact position of the stimulus and thus has the defining characteristics of complex cells in V1. The last two eigenvectors, which correspond to stimuli that minimize the quadratic term, are Gabor wavelets with an orientation orthogonal to that of the first two. As a consequence, the output of the quadratic term is inhibited by stimuli with an orientation orthogonal to the preferred one. In the case of Unit 28, the first and the last two eigenvalues have the same orientation but occupy two different halves of the RF. This means that Unit 28 is end inhibited; i.e., an extension of the length of an optimally oriented sine grating beyond the excitatory half of the RF into the inhibitory half leads to a decrease in response as opposed to a saturation as in conventional cells.

A direct interpretation of the remaining eigenvectors in the two units is difficult, although the magnitude of the eigenvalues shows that some of them elicit a strong response. Moreover, the interaction of the linear and quadratic terms to form the overall output of the quadratic form is not considered but cannot be neglected in general ${ }^{5}$ (even though it is small for the two units considered here). We show below that the optimal stimuli and the respective invariances can be more easily visualized and interpreted when the linear term is taken into account.

\section{Step 3}

Figure 2 shows the optimal excitatory and inhibitory stimuli of some of the units in the simulation. In almost all cases, $\mathbf{x}^{+}$ looks similar to a Gabor wavelet, in agreement with physiological data for neurons in V1 (ref. 22). $\mathbf{x}^{-}$is usually structured as well, which suggests that inhibition also plays an important role in shaping the response of the unit. As in these two cases the linear term is negligible ${ }^{20}$, the optimal stimuli of Units 4 and 28 are almost identical to the eigenvectors of largest magnitude (compare Fig. 1, first and last eigenvectors, with Fig. 2, Units 4 and 28). However, for quadratic forms with a significant linear term this does not need to be the case.

\section{Steps 4-7}

Figure 4 shows animations corresponding to some representative invariances. The animations show that the behavior of the units is more complex than suggested by the eigenvectors alone. They have active mechanisms to shape their orientation and frequency bandwidths and some have a limited invariance to changes in curvature (this characteristic is often associated with 
end or side inhibition). Figure 5a shows the distribution of 50,000 independent second derivatives of the invariances of random quadratic forms and that of the first 50 units learned in the simulation. The latter is clearly skewed toward zero (i.e., toward more invariant directions) and has a peak near zero. Of all invariances, $28 \%$ were classified as significant according to the $95 \%$ confidence interval for the random quadratic forms (dashed line). Figure $5 \mathbf{b}$ shows the number of significant invariances for the individual units (each unit has 49 invariances in total). Of 50 units, 46 have three or more significant invariances. The first invariance corresponds in all cases to a phase-shift invariance and was always classified as highly significant $(P<0.0005)$, which confirms that the units behave in a similar way to complex cells.

ACKNOWLEDGMENTS The figures have been reproduced with permission from ref. 20. This work has been supported by a grant to L.W. from the Volkswagen Foundation and by the Gatsby Charitable Foundation.

COMPETING INTERESTS STATEMENT The authors declare that they have no competing financial interests.

Published online at http://www.natureprotocols.com

Reprints and permissions information is available online at http://npg.nature.com/ reprintsandpermissions

1. Berkes, P. \& Wiskott, L. Applying slow feature analysis to image sequences yields a rich repertoire of complex cell properties. In Artificial Neural Networks-ICANN 2002 Proceedings, Lecture Notes in Computer Science (ed. Dorronsoro, J.R.) 81-86 (Springer, Berlin, 2002).

2. Berkes, P. \& Wiskott, L. Slow feature analysis yields a rich repertoire of complex cell properties. J. Vis. 5, 579-602 (2005).

3. Marmarelis, P. \& Marmarelis, V. Analysis of physiological systems: the white-noise approach (Plenum Press, New York, 1978).

4. de Ruyter van Steveninck, R. \& Bialek, W. Real-time performance of a movementsensitive neuron in the blowfly visual system: coding and information transfer in short spike sequences. Proc. R. Soc. Lond. B Biol. Sci. 234, 379-414 (1988).

5. Lewis, E.R., Henry, K.R. \& Yamada, W.M. Tuning and timing in the gerbil ear: Wiener-kernel analysis. Hear. Res. 174, 206-221 (2002).

6. Touryan, J., Lau, B. \& Dan, Y. Isolation of relevant visual features from random stimuli for cortical complex cells. J. Neurosci. 22, 10811-10818 (2002).

7. Rust, N.C., Schwartz, 0., Movshon, J.A. \& Simoncelli, E.P. Spike-triggered characterization of excitatory and suppressive stimulus dimensions in monkey V1. Neurocomp. 58-60, 793-799 (2004).

8. Simoncelli, E.P., Paninski, L., Pillow, J.W. \& Schwartz, 0. Characterization of neural responses with stochastic stimuli. In The Cognitive Neurosciences 3rd edn. (ed. Gazzaniga, M.S.) 327-338 (MIT Press, Cambridge, Massachusetts, 2004).
9. Rust, N.C., Schwartz, 0., Movshon, J.A. \& Simoncelli, E.P. Spatiotemporal elements of macaque V1 receptive fields. Neuron 46, 945-956 (2005).

10. Touryan, J., Felsen, G. \& Dan, Y. Spatial structure of complex cell receptive fields measured with natural images. Neuron 45, 781-791 (2005).

11. Rapela, J., Mendel, J.M. \& Grzywacz, N.M. Estimating nonlinear receptive fields from natural images. J. Vis. 4, 441-474 (2006).

12. Schwartz, 0., Pillow, J.W., Rust, N.C. \& Simoncelli, E.P. Spike-triggered neural characterization. J. Vis. 6, 484-507 (2006).

13. Franz, M.0. \& Schölkopf, B. A unifying view of Wiener and Volterra theory and polynomial kernel regression. Neural Comput. 18, 3097-3118 (2006).

14. Hashimoto, W. Quadratic forms in natural images. Netw. Comput. Neural Syst. 14 765-788 (2003).

15. Bartsch, H. \& Obermayer, K. Second-order statistics of natural images. Neurocomp. 52-54, 467-472 (2003).

16. Hyvärinen, A. \& Hoyer, P. Emergence of phase- and shift-invariant features by decomposition of natural images into independent feature subspaces. Neural Comput. 12, 1705-1720 (2000).

17. Hyvärinen, A. \& Hoyer, P. A two-layer sparse coding model learns simple and complex cell receptive fields and topography from natural images. Vision Res. 41, 2413-2423 (2001).

18. Körding, K.P., Kayser, C., Einhäuser, W. \& König, P. How are complex cell properties adapted to the statistics of natural stimuli? J. Neurophysiol. 91, 206-212 (2004).

19. Adelson, E.H. \& Bergen, J.R. Spatiotemporal energy models for the perception of motion. J. Opt. Soc. Am. A 2, 284-299 (1985).

20. Berkes, P. \& Wiskott, L. On the analysis and interpretation of inhomogeneous quadratic forms as receptive fields. Neural Comput. 18, 1868-1895 (2006).

21. Wiskott, L. \& Sejnowski, T.J. Slow feature analysis: unsupervised learning of invariances. Neural Comput. 14, 715-770 (2002).

22. Jones, J.P. \& Palmer, L.A. An evaluation of the two-dimensional Gabor filter model of simple receptive fields in cat striate cortex. J. Neurophysiol. 58, 1233-1257 (1987). 